\title{
An analysis of measurement from a 3D oceanic wave field
}

\author{
P. C. $\mathrm{Liu}^{1}$, C. H. Wu ${ }^{2}$, K. R. MacHutchon ${ }^{3}$ \& D. J. Schwab ${ }^{1}$ \\ ${ }^{I}$ NOAA Great Lakes Environmental Research Laboratory, USA \\ ${ }^{2}$ Department of Civil and Environmental Engineering, \\ University of Wisconsin-Madison, USA \\ ${ }^{3}$ Liebenberg \& Stander International (Pty) Ltd, South Africa
}

\begin{abstract}
We present here a preliminary examination and analysis of a small suite of 3D wave data to explore what new insight or inference we can garner - particularly toward the realm where conventional approaches have not yet been. While we caught a few glimpses that might indicate a need for new conceptualizations, it by no means negates the vast positive contributions that the conventional approaches have allowed us to make in the past century. We feel it is timely to encourage further 3D ocean wave measurement and thereby facilitate fresh new states of study and to enhance our understanding of ocean waves.
\end{abstract}

Keywords: wind waves, $3 D$ wave measurements, ocean waves, wave data analysis.

\section{Introduction}

We consider here the configuration of ocean waves to be a three-dimensional phenomenon in the sense that it is a function of $(x, y, z, t)$ or more specifically, $\mathrm{z}$ $=\mathrm{f}(\mathrm{x}, \mathrm{y}, \mathrm{t})$. Note that conventional time series wave measurement at a fixed single point, $\mathrm{z}=\mathrm{f}\left(\mathrm{x}_{0}, \mathrm{t}\right)$, is basically a function of one-dimensional surface fluctuation at a given single point, $\mathrm{x}_{0}$, with respect to time $\mathrm{t}$. Incidentally data of the case $z=f\left(x, t_{0}\right)$, which is in essence a snapshot of an ocean segment at a single time point, $\mathrm{t}_{0}$, with respect to an one-dimensional direction $\mathrm{x}$, is also a single point measurement.

So for over six decades, the ocean wave research community has been content with a general perception of ocean waves that was predominantly based on 
single point in-situ wave measurement, $\left(\mathrm{x}_{0}, \mathrm{t}\right)$, or $\left(\mathrm{x}, \mathrm{t}_{0}\right)$, either Eulerian from fixed probes or Lagrangian from floating instruments. As a result, the present day conventional conceptualization of ocean wave studies has been strictly $\left(\mathrm{x}_{0}, \mathrm{t}\right)$ oriented, with seemingly three-dimensional dynamics and models built around it. It has thus forged a kind of subjective reality for which whole ocean wave processes are described through this one-dimensional single-point realization of conventional wave measurements.

But the need for more realistic ocean wave measurements is gradually being recognized. At the recent OMAE 2008 Conference, there were at least two separate presentations, by Liu et al. [3] and Gallego et al. [1], independently advocating non-intrusive stereo imaging measurement with three and two digital video cameras systems respectively. The technology of digital cameras has advanced by leaps and bounds in recent years. And at the same time, the study of wind waves and wave modelling, actuated through five decades of singlepoint wave measurements, may have been "reaching a cul-de-sac, yielding 'no more great revelations or revolutions, but only incremental, diminishing returns" "as Horgan [2] regarded as the predicament of general science over a decade ago.

The ultimate goal of the development of these new stereo measurement systems is undoubtedly to provide three-dimensional wave surface fluctuations with respect to time, $z=f(x, y, t)$. Hopefully this new approach will become the mainstay of wave measurement and analysis studies and replace the traditional one-dimensional single-point time-series data analysis method. Provided, of course, we can manage to rise above our deeply seated, familiar comfort zone of one-dimensional mentality.

\section{Before single point wave measurement}

Nearly two decades before the advent of conventional single-point wave measurements in the mid-1940's, and prior to the start of the present-day use of pressure cell, step-resistance staff, or buoy accelerometer wave measurements that are all confined at a single location, early $20^{\text {th }}$ century attempts at measuring ocean waves were focused mainly on a broader area of the actual ocean such as using stereophotogrammetry as described in Sverdrup et al. [4]. An example given in the book is shown in Figure 1.

Considering that the contour results were made long before conventional wave spectrum conceptualization and single point wave measurements, it is certainly a remarkable accomplishment in the early part of the last century. What is also interesting in this topographic contour plot is the $9 \mathrm{~m}$ high point in the centre as compared with the $1 \mathrm{~m}$ low point in the upper right corner, a rising of $8 \mathrm{~m}$ in elevation and $57 \mathrm{~m}$ in horizontal distance. While we may not now be surprised by the appearance of the contour picture, Sverdrup et al. [4] did comment about the striking irregularity in the topography. Nevertheless it is an extraordinary snapshot of a portion of the ocean surface for its time. 


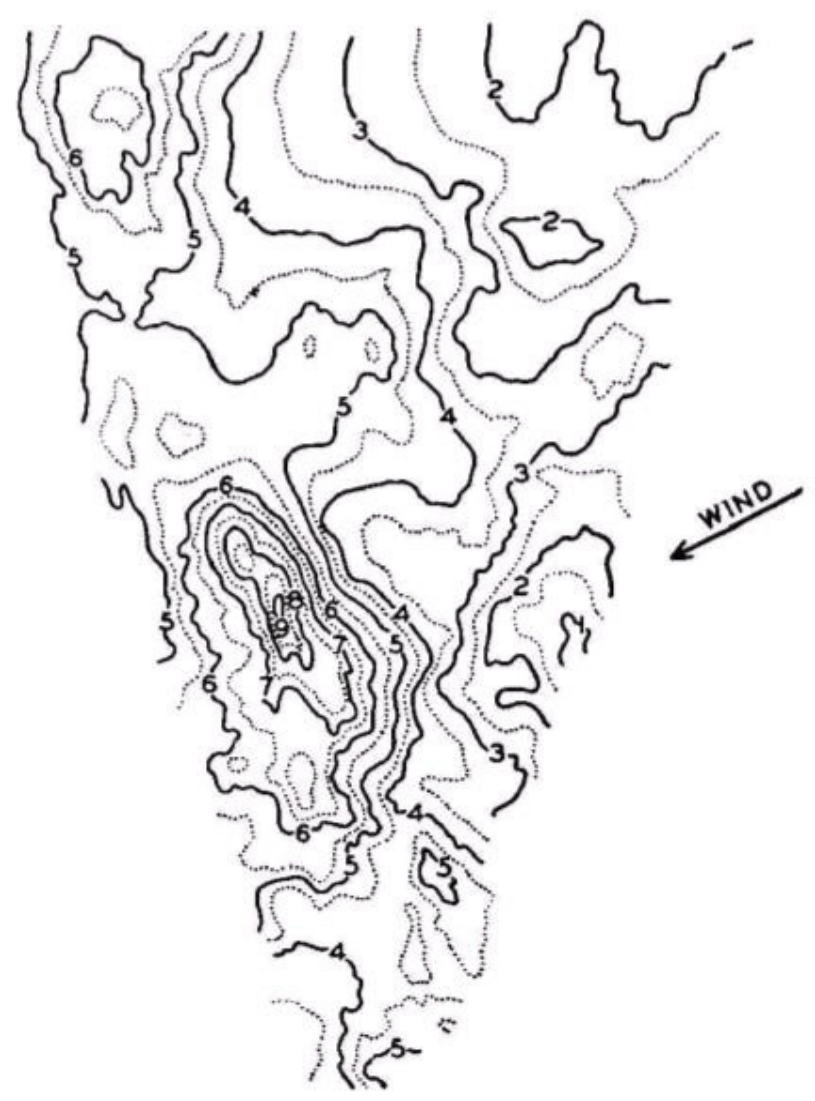

Figure 1: Topography of the sea surface derived from stereophotogrammetry of the sea surface taken onboard Meteor on January 23, 1926. From Sverdrup et al. [4].

\section{The ATSIS measurement}

Now fast forward some eight decades, where technology advances have made possible the use of digital video cameras for ocean wave measurement. Warnek and $\mathrm{Wu}$ [5] developed the Automated Trinocular Stereo Imaging System (ATSIS) for non-intrusively measuring the temporal evolution of threedimensional wave characteristics. The ATSIS system can provide a contour picture of the ocean surface every fraction of a second or more, depending on whatever resolution is required. So while the idea of stereo 3D imaging of the ocean surface is not exactly new, the emerging state of the art ATSIS system could provide measurement of 3D ocean wave fields with respect to time. It is certainly a new arena we are only starting to explore.

For those of us who were brought up during the era where single point wave measurement was the only practice available and have been conditioned to 
believe that a wave spectrum is an embodiment of the whole ocean wave process, the 3D imaging and the resulting pertinent and profuse data will be clearly overwhelming. But the new system will also be capable of unlocking a whole new range of possibilities for ocean wave studies, invigorating a field that has been stagnant for quite some time.

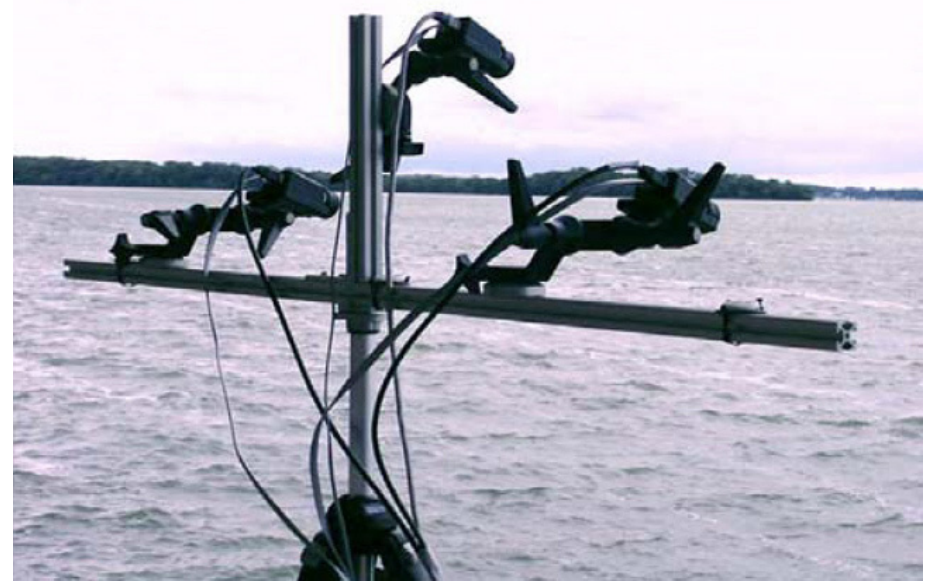

Figure 2: The three camera set up for the ATSIS system deployed in the field.

\section{Data from a 3D wave field}

So the data available is in general,

$$
\eta=f\left(x_{0}, t\right)
$$

for the conventional single point wave measurement; and

$$
\eta=f(x, y, t)
$$

for the new 3D measurement. As the measurement of eqn. (1) typifies the familiar time series, an example of the measurement of eqn. (2) at $t=t_{0}$, on the other hand, is shown in Figure 3 as the topography of the ocean surface.

As the actual wave measurement from the ATSIS system is a video recording not a single image, the full extent cannot be shown in print form. But in the age of internet, a portion of the measured video used in this study has been posted and can be seen on the internet at http://www.youtube.com/ watch? $=$ pMYENsrSLN4.

Now the digital data used in this study, as derived from the video recording, is a three dimensional $(x, y, \eta)$ data set of [441x251x151] coordinates, that in essence represents $15 \mathrm{sec}$ of data with $x$ and $y$ coordinates that remain the same for each time step, while the $\eta$ coordinate varies with respect to $(x, y, t)$, sampled at a frequency of $10 \mathrm{~Hz}$. 


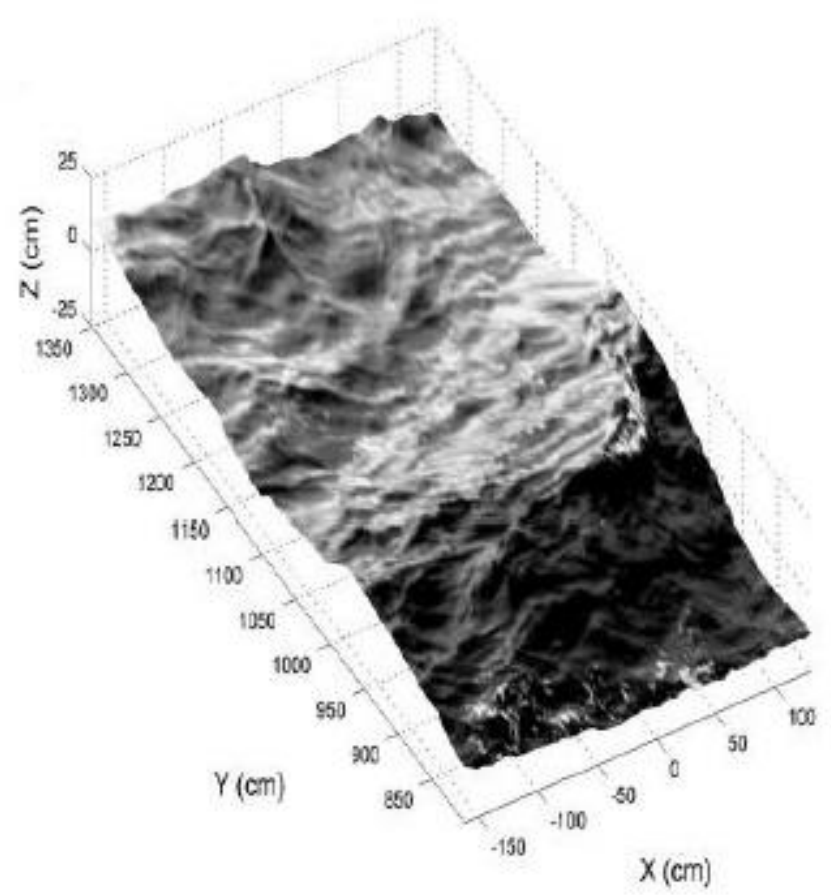

Figure 3: $3 \mathrm{D}$ topography of a portion of the ocean surface at a given instant of time.

\section{A grappling with 3D wave data analysis}

To some extent, viewing the afore-mentioned video recording is no different than watching the ocean surface from a cruise ship out there in the deep ocean (e.g. http://www.youtube.com/watch? $\mathrm{v}=$ MnoTj7Jx4L4.) Only now we are capable of making more realistic wave measurements. The key question to ask is really: what would be the pertinent course to follow to analyze this new wealth of data?

Undoubtedly new approaches will continue to evolve as more data become available. In the mean time, we are confronted with the availability of 15 seconds of $(x, y, \eta, t)$ data, that was recorded in a small lake, specifically Lake Mendota, near Madison, Wisconsin. To proceed, we shall start with an exploratory approach by performing conventional analysis for each individual single data point. For a pixel grid of $441 \mathrm{x} 251$, the data is in fact equivalent to having 110,691 single points with each point providing time series data for 15 seconds with $10 \mathrm{~Hz}$ resolution. To effectively visualize all these volumetric data, we simply calculate the total energy represented by each individual standard deviation wave height, i.e., $4 *$ standard deviation, to demonstrate their essential 
character and then plot them in 3D space as shown in Figure 4. It is rather surprising and pleasant to see this well formed result from basically a statistical estimate for each pixel point. Furthermore the distribution of the standard deviation of the wave heights is shown by the histogram in Figure 5. One might be tempted to try fitting a distribution function to this clearly skewed case. We do not feel that really serves much meaningful purpose. Suffice it to say that in the 3D framework, we can readily obtain useful information using only $15 \mathrm{sec}$ data. This is inconceivable in the conventional framework.

Now with the variations exhibited, an immediate question is: what is the wave height representative in this $3 \mathrm{D}$ wave field?

Being completely nurtured in the conventional conceptualization, we are conditioned to regard a wave height as the distance between the trough and crest in a single location. This is very perceptive and straightforward when we look at a customary plot of time series data at a single location. But in the open ocean, how do we sift through a distance between a crest and a trough? So what is the wave height for a given region of the ocean?

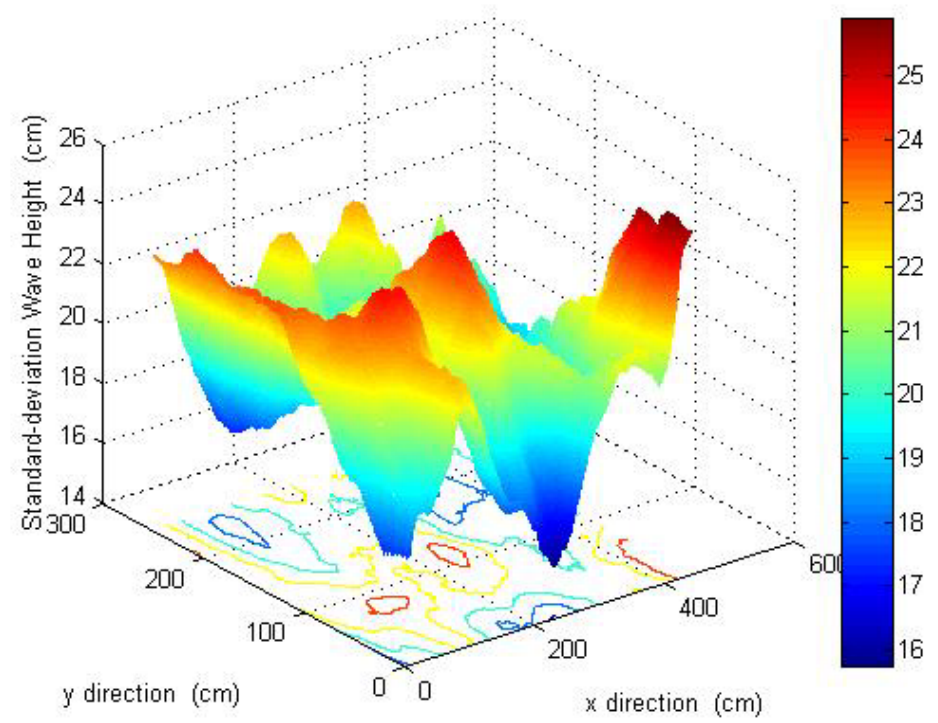

Figure 4: A 3D plot of the standard deviation wave heights for the pixels, each represents a single point data set.

The conventional practice of using one single-point significant wave height to represent the wave height of a region of the ocean surface is clearly no longer valid in a $3 \mathrm{D}$ wave field. Conceivably when a seafarer in the open ocean talks about a wave height, it is most likely the height of a visible crest rather than something between trough and crest. So we choose to first examine the highest crest and lowest trough at each instance of the data set. 


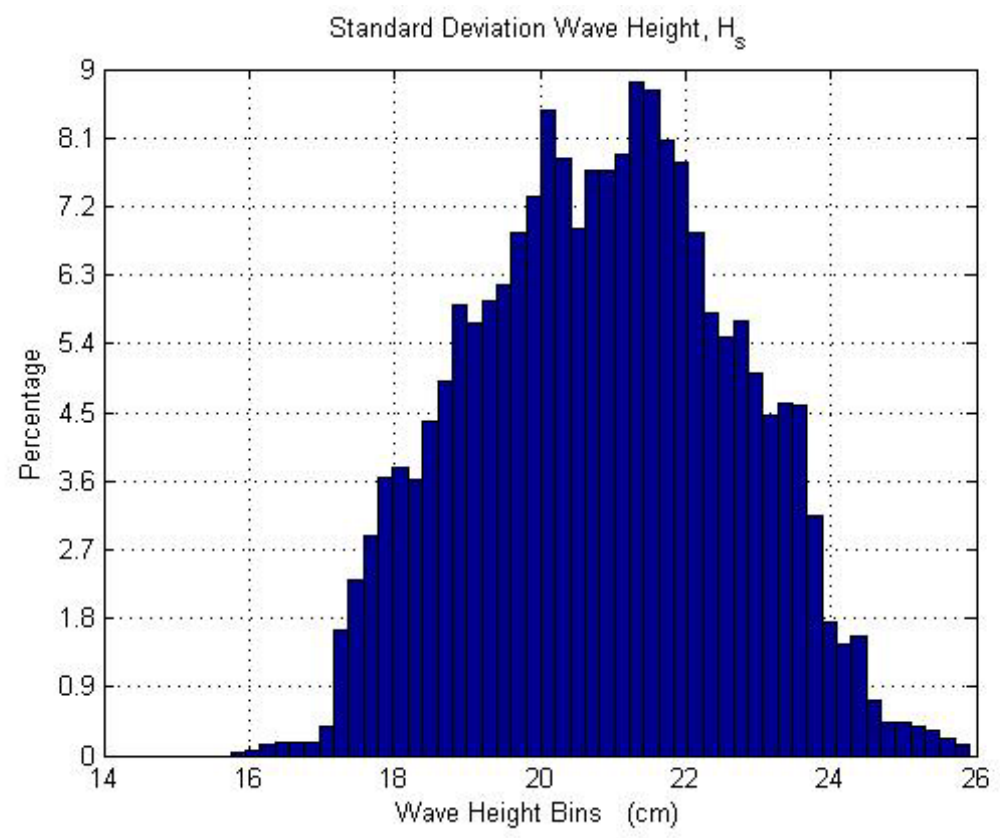

Figure 5: $\quad$ Histogram of the standard deviation wave height given in Figure 4.

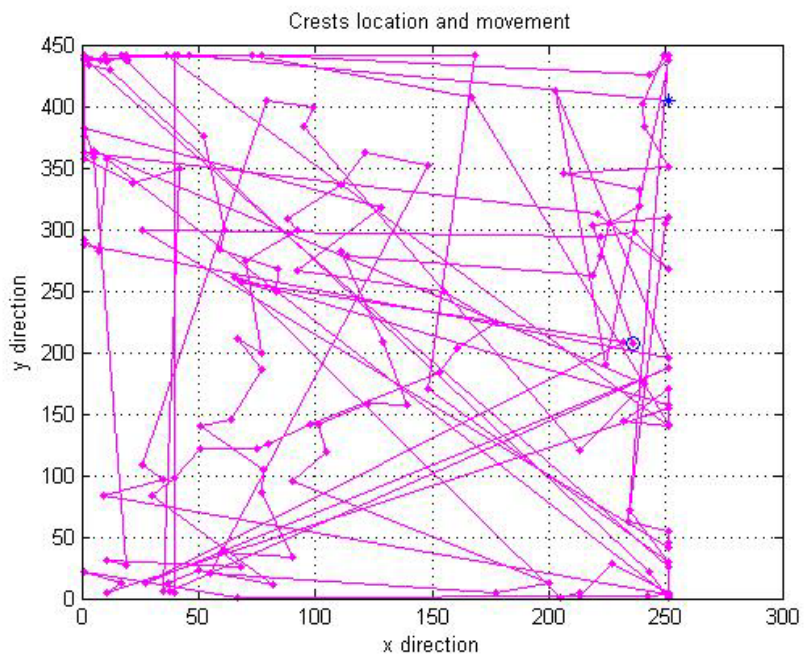

Figure 6: The crest locations of the data set. The starting and ending locations in time are marked by an * and a circle respectively. 


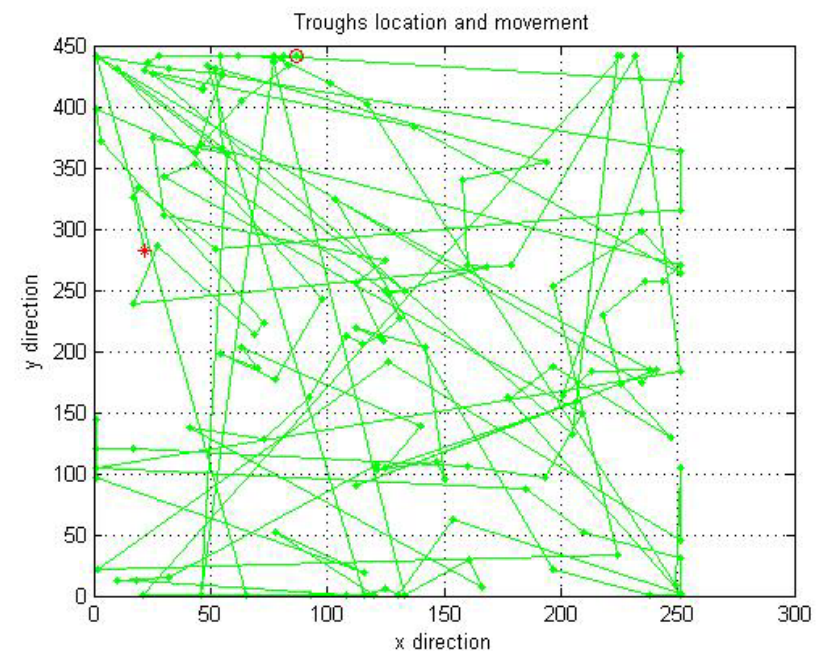

Figure 7: The trough locations of the data set. Again the starting and ending locations in time are marked by an * and a circle respectively.

As shown in Figures 6 and 7, the crest and the trough, being the highest and lowest points at each instance, move around constantly, but they never occur at the same location at the same instance. Thus it is understandable that the familiar notion of wave height evolved from elementary trigonometry and time series analysis cannot be generalized to the 3D wave field as one might wish to bring it into play.

As a matter of fact, if we connect from the trough to crest at each instant, and then connect the crest to the trough of the next instant, and repeat the process throughout the whole data set, the result is Figure 8. It is interesting to note that the points are fairly evenly spread around the region, but none is really on top of each other.

Alternatively we also plotted the crests, troughs, and the sums of corresponding crest and trough, with respect to time as shown in Figure 9. It gives us some indication of the surface fluctuations of the ocean surface in that region. This is aimed at practical reference, which may or may not be meaningful. But the question regarding what is the wave height in a $3 \mathrm{D}$ wave field remains unanswered.

Finally, we have also tried to examine the possibility of calculating the wave number spectra for each instant of the data set. For the instant surfaces shown in Figure 10, their corresponding wave number spectra are given in Figure 11. Because the data only covered $15 \mathrm{sec}$, it may not have sufficient oscillations to provide transient processing and consequential interpretations. At any rate, it is only an indication of what can be done with the data set. Under the conventional approach, a $15 \mathrm{sec}$ measurement will certainly not provide any information about the underlying wave process. 


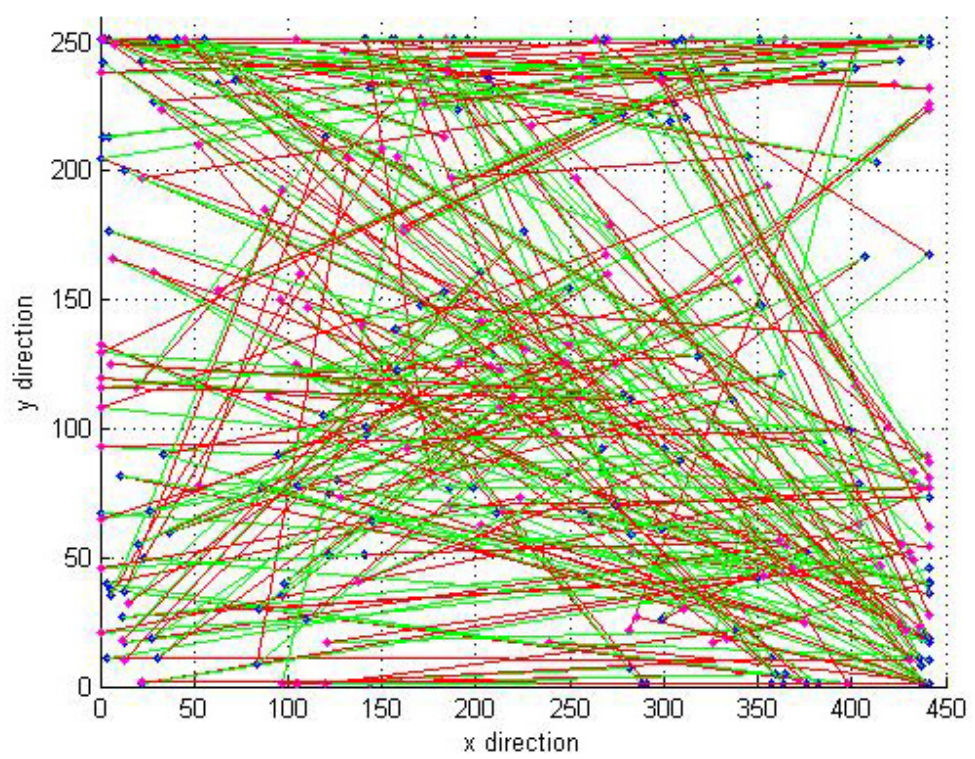

Figure 8: Connecting the trough of each instant to the crest, in dark grey, and connecting the crest to the trough of the next instant, in light grey, and repeating the process throughout the data set.

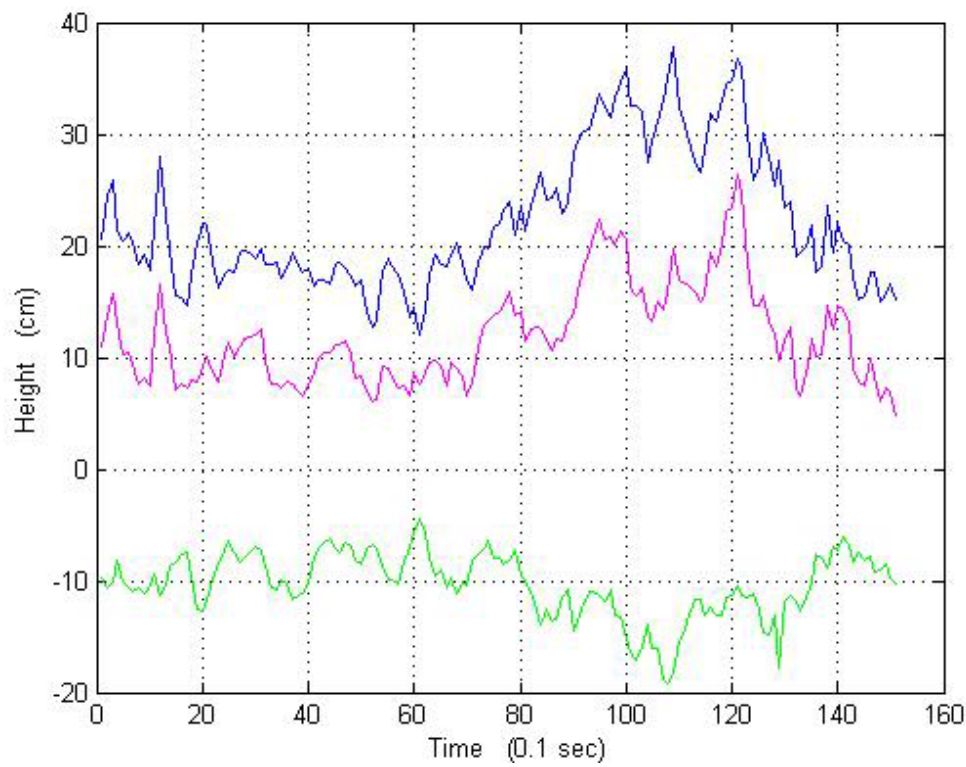

Figure 9: Plot of the height of crest (middle), trough (bottom), and the sum of crest + trough (top) with respect to time. 


\section{Coastal Processes}

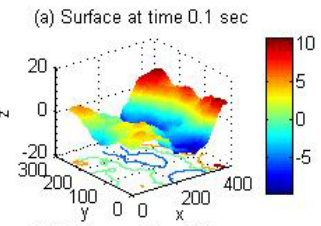

(c) Surface at time $0.5 \mathrm{sec}$

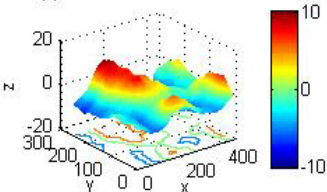

(e) Surface at time $0.9 \mathrm{sec}$

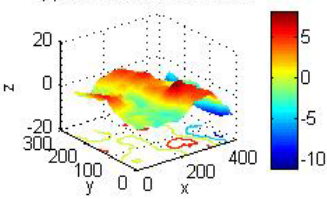

(b) Surface at time $0.3 \mathrm{sec}$

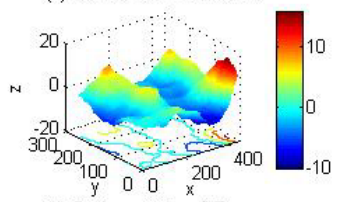

(d) Surface at time $0.7 \mathrm{sec}$

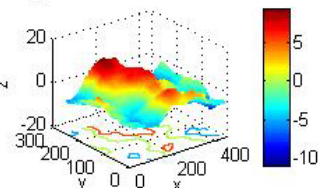

(f) Surface at time $1.1 \mathrm{sec}$

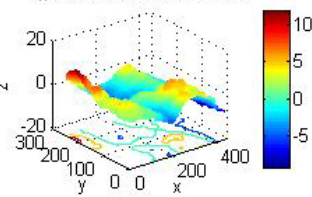

Figure 10: Examples of recorded instant wave surface during the first second of measurement.
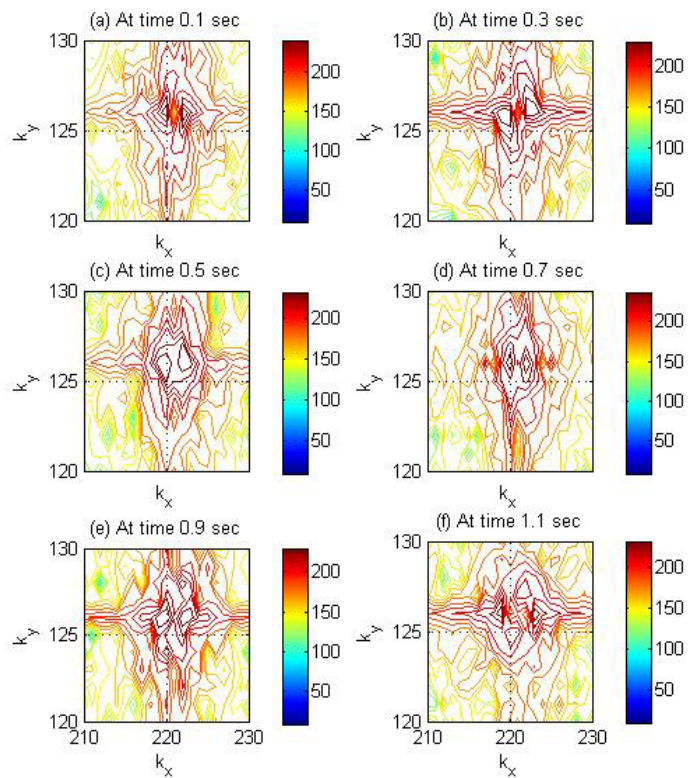

Figure 11: Wave number spectra for the corresponding surfaces given in Figure 10. 
All of these analyses presented here are tentative. One of the basic premises of the conventional conviction on the long standing single location ocean wave measurement is that the data from this single location measurement represents the wave condition of a general region. So if waves are measured from another point of this region, it should be generally the same. We found that is not the case. Even for a small pixel region the data are vary from one pixel to the next. Herein lies the answer to why Komen et al. [6] were not able to make wave predictions that always fall within the error bands of the observations as expected. Single-point in-situ wave measurement is simply incapable of embodying the realistic ocean waves for more than just that one single-point location. The theoretically-refined wave prediction model cannot be made in accord with the observations at a single point because the single point observation does not represent the reality that the theoretical model is trying to portray. It cannot be over stressed that we need the more comprehensive ATSIS 3D ocean wave measurement.

\section{Concluding remarks}

After six decades of dominating ocean wave conceptualization as the innate reality, single-point wave measurements have served well the general wave studies. However the progress and model refinement has been in stagnate during the last dozen years or more, it is timely that a new system of ocean wave measurement should be initiated and implemented. Along with this new frontier of ocean wave data measurement, there will be a whole new realm of wave data analysis. New paradigms and new conceptualizations that have not yet been contemplated should be further explored. For instance, instead of the distance between a trough and an adjacent crest at a single point, what should a wave height in the three-dimensional wave field be? There should never be any shortage of incentive or excitement in this new ocean wave measurement frontier. It is time to open up to fresh new perspectives and ideas.

\section{Acknowledgement}

This is GLERL Contribution No. 1512.

\section{References}

[1] Gallego, G., A. Benetazzo, A. Yezzi, and F. Fedele, 2008: Wave statistics and spectra via a variational wave acquisition stereo system, Proceedings, OMAE2008, Portugal.

[2] Horgan, J., 1996: The End of Science, Addison-Wesley.

[3] Liu, P. C., D. J. Schwab, C. H. Wu, and K. MacHutchon, 2008: Wave heights in a 4D ocean wave field, Proceedings, OMAE2008, Portugal.

[4] Sverdrup, H. U., M. W. Johnson, and R. H. Fleming, 1942: The Oceans, their physics, chemistry, and general biology. Prentice-Hall, Inc., 1087p. 
[5] Wanek, J. M. and C. H. Wu, 2006: Automated trinocular stereo imaging system for three-dimensional surface wave measurements. Ocean Engineering, vol. 33, 723-747.

[6] Komen, G.J., L. Cavaleri, M. Donelan, K. Hasselmann, S. Hasselmann, and P.A.E.M. Janssen, 1994: Dynamics and Modelling of Ocean Waves, Cambridge, 532p. 